\title{
Collimated UV light generation by two-photon excitation to a Rydberg state in $\mathbf{R b}$ vapor
}

\author{
Mark Lam ${ }^{1}$, Sambit B. Pal ${ }^{1}$, Thibault Vogt ${ }^{1,2}$, Christian Gross $^{1}$, Martin Kiffner ${ }^{1,3}$, And \\ WENHUI LI $1,4{ }^{*}$ \\ ${ }^{1}$ Centre for Quantum Technologies, National University of Singapore, 3 Science Drive 2, Singapore 117543 \\ ${ }^{2}$ MajuLab, CNRS-UNS-NUS-NTU International Joint Research Unit UMI 3654, Singapore 117543 \\ ${ }^{3}$ Clarendon Laboratory, University of Oxford, Parks Road, Oxford OX1 3PU, United Kingdom \\ ${ }^{4}$ Department of Physics, National University of Singapore, Singapore 117542 \\ *Corresponding author: wenhui.li@nus.edu.sg \\ Compiled March 14, 2019
}

We use two continuous-wave (CW) laser beams of 780 $\mathrm{nm}$ and $515 \mathrm{~nm}$ to optically drive ${ }^{85} \mathrm{Rb}$ atoms in a heated vapor cell to a low-lying Rydberg state $10 \mathrm{D}_{5 / 2}$. We observe a collimated ultraviolet (UV) beam at $311 \mathrm{~nm}$, corresponding to the transition frequency from the $\mathbf{1 1 P}_{3 / 2}$ state to the $5 S_{1 / 2}$ state. This indicates the presence of a coherent four-wave mixing process, built up by two input laser fields as well as a terahertz $(\mathrm{THz})$ radiation of $3.28 \mathrm{THz}$ that is generated by amplified spontaneous emission between the $10 \mathrm{D}_{5 / 2}$ and the $11 \mathrm{P}_{3 / 2}$ states. We characterize the $311 \mathrm{~nm}$ UV light generation and its dependence on various physical parameters. This scheme could open up a new possibility for generating narrowband THz wave as well as deep UV radiation.

(C) 2019 Optical Society of America

OCIS codes: $\quad$ (020.1670), (020.5780), (300.6210).

http://dx.doi.org/10.1364/ao.XX.XXXXXX

Laser-induced coherence among quantum states in an atomic medium leads to greatly enhanced nonlinear susceptibilities compared to that of solid state crystal media [1], and gives rise to fascinating phenomena such as electromagnetically induced transparency (EIT) [2], coherent population trapping (CPT) [3], and lasing without inversion (LWI) [4]. Nonlinear optical processes in such coherently prepared atomic media have been extensively studied for a wide range of applications. In particular, four-wave mixing (FWM) in alkali atomic gases is used to produce entangled states of light [5] and photon pairs [6], to construct quantum memories [7], and to transfer orbital angular momentum between near-infrared and blue light fields [8]. A very active area of research on FWM has been the investigation of frequency up and down conversion by using continuous wave excitation lasers in hot atomic vapors, which enables the efficient generation of new coherent optical fields in the visible and infrared regions [9-18].

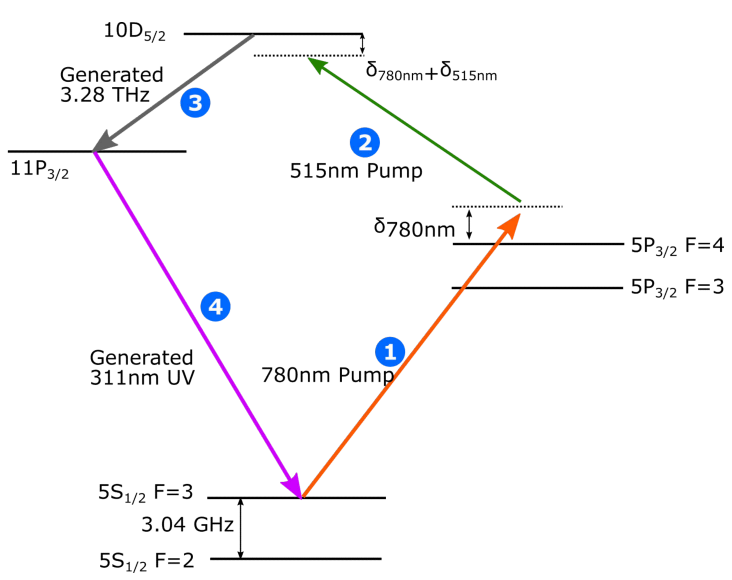

Fig. 1. Energy levels of ${ }^{85} \mathrm{Rb}$ relevant to our scheme. $\delta_{780 \mathrm{~nm}}$ is the detuning of the $780 \mathrm{~nm}$ pump laser from the $5 \mathrm{~S}_{1 / 2} \mathrm{~F}=3$ $\rightarrow 5 \mathrm{P}_{3 / 2} \mathrm{~F}=4$ transition resonance, while $\delta_{780 \mathrm{~nm}}+\delta_{515 \mathrm{~nm}}$ is the two-photon detuning from the resonance frequency between the $5 S_{1 / 2} F=3$ and $10 D_{5 / 2}$ states. The detunings are defined to be the laser frequency minus the resonance frequency. Numeric labels have been assigned to each transition for the reader's convenience.

In this paper, we investigate the generation of a collimated $\mathrm{UV}$ light in a ${ }^{85} \mathrm{Rb}$ thermal atomic vapour via a parametric fourwave mixing process involving the low-lying Rydberg states $10 \mathrm{D}_{5 / 2}$ and $11 \mathrm{P}_{3 / 2}$. The relevant energy levels and the corresponding transitions of the experiment are shown in Fig. 1. The two transitions, $5 \mathrm{~S}_{1 / 2} \rightarrow 5 \mathrm{P}_{3 / 2}$ (transition 1 ) and $5 \mathrm{P}_{3 / 2} \rightarrow 10 \mathrm{D}_{5 / 2}$ (transition 2), are driven by two near-resonant laser fields at $780 \mathrm{~nm}$ and $515 \mathrm{~nm}$, respectively. We experimentally observe a collimated and single wavelength laser beam at $311 \mathrm{~nm}$, which corresponds to the $11 \mathrm{P}_{3 / 2} \rightarrow 5 \mathrm{~S}_{1 / 2}$ transition (transition 4 in Fig. 1). We attribute this generated $311 \mathrm{~nm}$ beam to a four-wave mixing process from the two input lasers as well as the $3.28 \mathrm{THz}$ radiation emitted on the $10 \mathrm{D}_{5 / 2} \rightarrow 11 \mathrm{P}_{3 / 2}$ transition (transition 3 in Fig. 1). The generation of this $\mathrm{THz}$ radiation via amplified 


\begin{tabular}{c|ccc|ccc|c}
\hline & \multicolumn{3}{|c|}{$10 \mathrm{D}_{5 / 2} \rightarrow n \mathrm{P}_{3 / 2}$} & \multicolumn{3}{c|}{$n \mathrm{P}_{3 / 2} \rightarrow 5 \mathrm{~S}_{1 / 2}$} & \\
\hline $\mathrm{n}$ & $v(\mathrm{THz})$ & $\lambda(\mathrm{nm})$ & $\left|d_{\mathrm{DP}}\right|\left(\mathrm{ea}_{0}\right)$ & $v(\mathrm{THz})$ & $\lambda(\mathrm{nm})$ & $\left|d_{\mathrm{PS}}\right|\left(\mathrm{ea}_{0}\right)$ & $\left|d_{\mathrm{DP}} \cdot d_{\mathrm{PS}}\right|^{2}\left(\left(\mathrm{ea}_{0}\right)^{4}\right)$ \\
\hline 11 & 3.3 & 91221.5 & 63.64 & 962.9 & 311.3 & 0.03 & 3.645 \\
10 & 17.0 & 17637.1 & 1.44 & 949.2 & 315.8 & 0.04 & 0.0033 \\
9 & 37.7 & 7950.0 & 1.24 & 928.5 & 322.9 & 0.05 & 0.0038 \\
8 & 71.2 & 4212.3 & 0.83 & 895.0 & 335.0 & 0.05 & 0.0017 \\
7 & 130.6 & 2294.6 & 0.55 & 835.5 & 358.8 & 0.10 & 0.0030 \\
6 & 252.9 & 1185.5 & 0.36 & 713.3 & 420.3 & 0.27 & 0.0095 \\
5 & 581.9 & 515.2 & 0.22 & 384.2 & 780.2 & 2.98 & 0.4298 \\
\hline
\end{tabular}

Table 1. Table of frequencies $(v)$, wavelengthes $(\lambda)$, and dipole matrix elements $\left(d_{D P}\right.$ and $\left.d_{P S}\right)$ for the transitions $10 \mathrm{D}_{5 / 2} \rightarrow n \mathrm{P}_{3 / 2}$ and $n \mathrm{P}_{3 / 2} \rightarrow 5 \mathrm{~S}_{1 / 2}$. Given in the last column are the values of $\left|d_{\mathrm{DP}} \cdot d_{\mathrm{PS}}\right|^{2}$. The $10 \mathrm{D}_{5 / 2} \rightarrow n^{\prime} \mathrm{F}$ transitions and further cascade transitions from $n^{\prime} \mathrm{F}$ states back to the $5 S_{1 / 2}$ state are not considered here.

spontaneous emission is facilitated by the long radiative lifetime of the $10 \mathrm{D}_{5 / 2}$ state $(\sim 780 \mathrm{~ns})$ [19], which enables population inversion respect to $11 \mathrm{P}_{3 / 2}$ with moderately strong pumping.

Note that a similar scheme involving the lower lying states $5 \mathrm{D}_{5 / 2}$ and $6 \mathrm{P}_{3 / 2}$ has been studied in several experiments using rubidium vapors [9-16], where a collimated blue light at 421 $\mathrm{nm}$, corresponding to the transition $6 \mathrm{P}_{3 / 2} \rightarrow 5 \mathrm{~S}_{1 / 2}$, is produced via a four-wave mixing process. A generated $5.2 \mu \mathrm{m}$ infrared radiation corresponding to the transition $5 \mathrm{D}_{5 / 2} \rightarrow 6 \mathrm{P}_{3 / 2}$, enabling the FWM process, has been observed in some of the experiments [14]. The difference between our scheme and the works in [9$16]$ is that, instead of the $5 D_{5 / 2}$ state, a low-lying Rydberg state is being excited by pump lasers. As a result, two very different frequencies outside the typical optical regime are generated, namely a THz field on transition 3 and a UV field on transition 4. In our current setup, the direct measurement of the generated $3.28 \mathrm{THz}$ field is hindered by the opacity of the fused-silica vapour cell windows at $\mathrm{THz}$ frequencies. However, the presence of the $\mathrm{THz}$ field is firmly established by the observation of the collimated $311 \mathrm{~nm}$ UV light.

Our experimental setup is illustrated in Fig. 2. Two pump lasers consist of a $780 \mathrm{~nm}$ Titanium Sapphire (Ti:Sa) laser driving transition 1 and a $515 \mathrm{~nm}$ external cavity diode laser (ECDL) driving transition 2 . The frequency of the $780 \mathrm{~nm}$ pump laser is determined via an optical beat note measurement with a $780 \mathrm{~nm}$ reference laser, which is locked on the $5 \mathrm{~S}_{1 / 2} \mathrm{~F}=3$ to $5 \mathrm{P}_{3 / 2} \mathrm{~F}=4$ transition. Meanwhile, the $515 \mathrm{~nm}$ pump laser is stabilized to a $515 \mathrm{~nm}$ reference laser via frequency offset locking [20]. We lock this $515 \mathrm{~nm}$ reference laser to the spectrum of a laddertype EIT transition formed with the $10 \mathrm{D}_{5 / 2}, 5 \mathrm{P}_{3 / 2}$ and $5 \mathrm{~S}_{1 / 2}$ states (transitions 1 and 2). The EIT is realized in our setup by overlapping the two reference lasers, both right-circularly polarized, in a counter-propagating configuration through a $\mathrm{Rb}$ vapour cell [21, 22].

The two pump beams are combined on a dichroic mirror and coupled into a photonic crystal fiber (PCF) to ensure a perfect spatial overlap. The output from the PCF is configured for right circular polarization and focused with a single achromatic lens, and then sent into a commercially-available UV fused silica vapour cell containing an isotopic mixture of rubidium per its natural abundance. This cell is housed in a temperaturestabilized copper assembly using heatpipes with bifilar windings of heating wire. This increases the coil distance from the cell location and consequently reduces the effect of stray magnetic fields. At the exit end of the cell, we block the transmitted input pump lights with the use of UV-bandpass interference filters, and measure the power of the generated light using a calibrated UV-enhanced photodiode (PD).

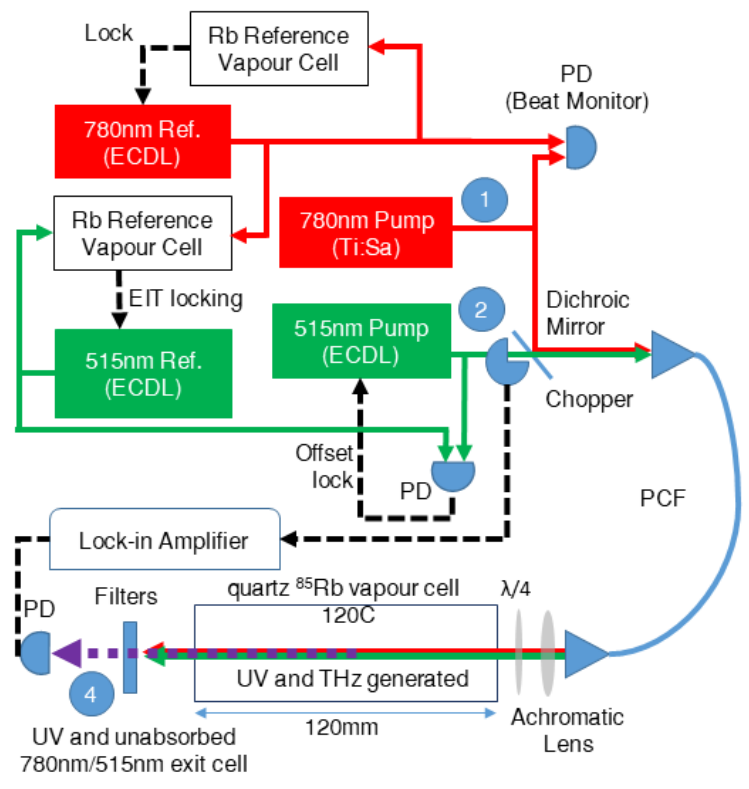

Fig. 2. Schematic representation of the experimental setup. The various optical paths have been assigned numeric labels matching their associated transitions as per Fig. 1. The cell temperature is regulated to $\sim 120^{\circ} \mathrm{C}$ for most measurements presented here. Details are given in the text.

We observe a UV beam at the exit of the vapor cell copropagating in the same direction as the input pump beams, over a range of experimental conditions. We first characterize the spatial profile of this UV beam by using the well-known knife-edge method [23, 24]. The measurement results in Fig. 3(a) show that the generated UV light and the input $515 \mathrm{~nm}$ beam overlap with each other, and have a Gaussian profile with nearly the same beam size. This directionality of the generated UV light is consistent with the phase-matching condition for fourwave mixing $\mathbf{k}_{1}+\mathbf{k}_{2}=\mathbf{k}_{3}+\mathbf{k}_{4}$, where $\mathbf{k}_{i}$ is the wave vector 

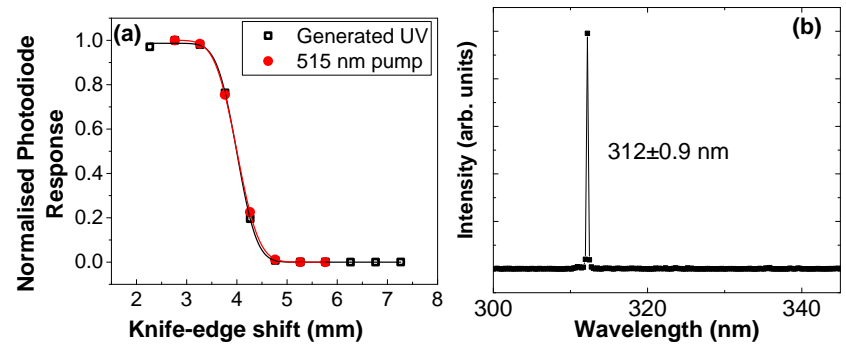

Fig. 3. (a) Knife-edge measurements of the generated UV light and the $515 \mathrm{~nm}$ pump light at $19 \mathrm{~cm}$ from the center of the vapour cell. The solid lines are the fits to the data with the error function, which is the integration of a Gaussian beam profile along the radial direction. (b) The spectrum of the output beam measured by a narrow-range grating spectrometer.

corresponding to transition $i(i \in\{1,2,3,4\})$.

The wavelength of the generated UV beam is measured with a grating spectrometer, and the only detectable peak is at $\sim 311$ $\mathrm{nm}$, as shown in Fig. 3 (b). This wavelength corresponds to the $11 \mathrm{P}_{3 / 2} \rightarrow 5 \mathrm{~S}_{1 / 2}$ transition. None of the other UV wavelengths listed in Table 1, corresponding to the transitions $n \mathrm{P}_{3 / 2} \rightarrow 5 \mathrm{~S}_{1 / 2}$ with $n \neq 10$, are visible on this power scale. We make the following simple arguments to explain this observation. The third order nonlinear susceptibility of FWM in atomic media is proportional to the product of the dipole matrix elements of the four corresponding transitions [2]. Since all the possible FWM pathways in Table 1 share the same two pumping transitions, the generated UV power of a FWM pathway, proportional to the square of the third order nonlinearity, will largely be determined by $\left|d_{\mathrm{DP}} \cdot d_{\mathrm{PS}}\right|^{2}$, where $d_{\mathrm{DP}}$ and $d_{\mathrm{PS}}$ are the dipole moments of the two cascade transitions. The values given in the last column of Table 1 indicate that the nonlinearity of the four-wave mixing via transitions 3 and 4 is much larger than that of other FWM pathways, which results in the domination of the $311 \mathrm{~nm}$ in the generated light.

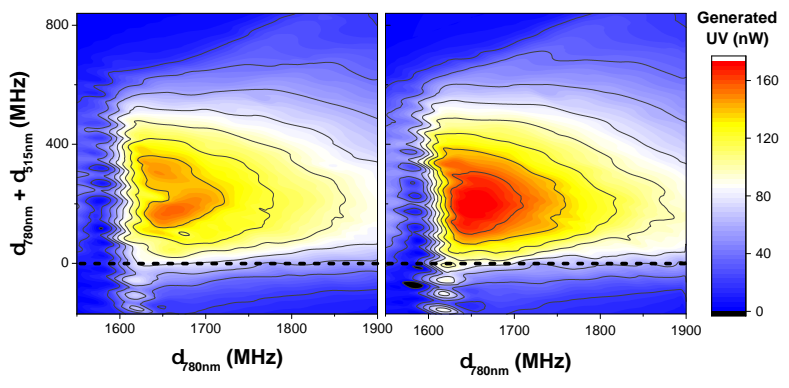

Fig. 4. Generated UV power as a function of the $780 \mathrm{~nm}$ pump laser detuning $\left(\delta_{780 \mathrm{~nm}}\right)$ and the two-photon detuning $\left(\delta_{780 \mathrm{~nm}}+\delta_{515 \mathrm{~nm}}\right)$ without the repump laser (left) and with the repump laser (right). The horizontal dashed line denotes the atomic two-photon resonance $\delta_{780 \mathrm{~nm}}+\delta_{515 \mathrm{~nm}}=0$. The cell temperature is $126^{\circ} \mathrm{C}$, while the $515 \mathrm{~nm}$ and $780 \mathrm{~nm}$ pump lasers have a power of $19 \mathrm{~mW}$ and $150 \mathrm{~mW}$, respectively.

The above spatial and spectral measurements confirm that this collimated UV beam is generated by four-wave mixing instead of spontaneous emission. Next we discuss the dependence of the UV generation on the two pump laser frequencies by scanning them independently. In Fig. 4 (a), the generated UV power is plotted as a function of the $780 \mathrm{~nm}$ detuning $\left(\delta_{780 \mathrm{~nm}}\right)$ and the two-photon detuning $\left(\delta_{780 \mathrm{~nm}}+\delta_{515 \mathrm{~nm}}\right)$. The optimal UV generation is observed near the two-photon resonance of transitions 1 and 2 with a width of around $250 \mathrm{MHz}$, which we attribute to Doppler broadening. Along the horizontal axis, an asymmetric peak appears with a sharp edge at around $\delta_{780 \mathrm{~nm}}=$ $1.65 \mathrm{GHz}$ and a long tail of width $\approx 800 \mathrm{MHz}$ for $\delta_{780 \mathrm{~nm}}>1.65$ $\mathrm{GHz}$. This feature can be understood as follows. We have experimentally verified that the strong Kerr-lensing near the resonance of transition 1 distorts the $780 \mathrm{~nm}$ pump beam, and renders the FWM process ineffective. The beam restores its original shape at a detuning $\delta_{780 \mathrm{~nm}} \sim 1.6 \mathrm{GHz}$, where the coherence of FWM survives and produces the $311 \mathrm{~nm}$ light that we observe. Note that a similar effect due to Kerr-lensing has been observed before in the generation of $421 \mathrm{~nm}$ light via FWM [12].

To check whether the optimal detuning of $\delta_{780 \mathrm{~m}} \sim 1.6 \mathrm{GHz}$ is related to the coherence induced by coupling to both hyperfine levels of the $5 S_{1 / 2}$ state [10], we repeat the spectroscopic measurements with an additional repumper ECDL locked on the $5 \mathrm{~S}_{1 / 2} \mathrm{~F}=2 \rightarrow 5 \mathrm{P}_{1 / 2} \mathrm{~F}=2$ transition of the $\mathrm{D} 1$ line. This repumper laser will redistribute most of the ground state population into the hyperfine level $5 S_{1 / 2} F=3$. Fig. 4 (b) shows that this repumper causes no shift in the optimal detuning compared to that in Fig. 4 (a), but results in an overall increase of $15 \%$ in the UV output. This result suggests that the UV generation observed is mostly due to the population in the $5 S_{1 / 2} F=3$ level.

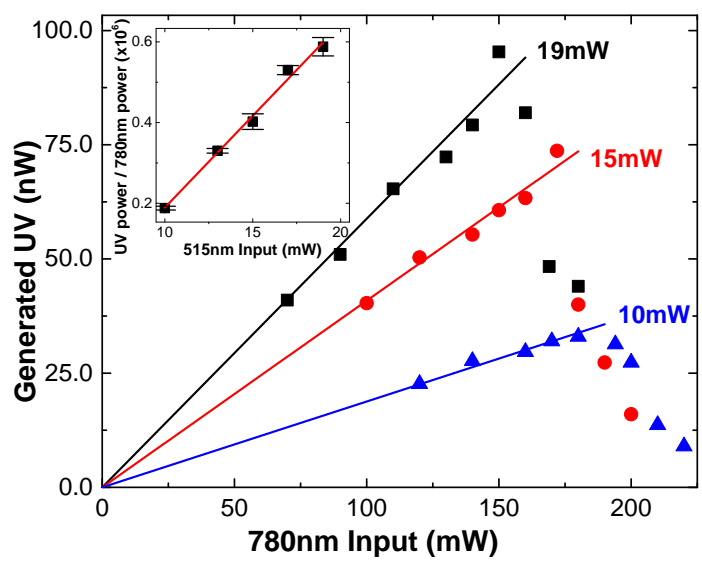

Fig. 5. Generated UV power as a function of the $780 \mathrm{~nm}$ pump power for the $515 \mathrm{~nm}$ pump powers of $19 \mathrm{~mW}$ (black square), $15 \mathrm{~mW}$ (red circle), and $10 \mathrm{~mW}$ (blue triangle). The data is taken at $123^{\circ} \mathrm{C}$. The solid lines are linear fits to the data of lower $780 \mathrm{~nm}$ powers, and the slopes of these fits with fitting errors are plotted as a function of the $515 \mathrm{~nm}$ power in the inset. The solid line in the inset is a linear fit.

We investigate the dependence of the generated UV light on the input laser powers, while keeping the detuning of both lasers at the values where maximum UV generation is observed. In Fig. 5, all curves show that the generated UV power first increases with increasing $780 \mathrm{~nm}$ power. A further increase of the $780 \mathrm{~nm}$ power results in a reduction of the UV generation. To better understand this dependence, we simulate the light propagation inside the vapor cell within a simple four-level model and semi-classical Maxwell-Bloch equations [25]. We find that the predicted UV power and its dependence on the pump powers agree qualitatively with the measurements. In particular, our simulations suggest that the shape of the curves in Fig. 5 is mostly determined by the population of the $10 D_{5 / 2}$ 
state as a function of the pump powers. In the inset of Fig. 5, the slopes of the linear fits at small $780 \mathrm{~nm}$ powers are shown to increase linearly with the $515 \mathrm{~nm}$ pump power, and has no sign of saturation within the range of $515 \mathrm{~nm}$ power available in our experiment. This indicates that using higher $515 \mathrm{~nm}$ power will improve the UV generation.

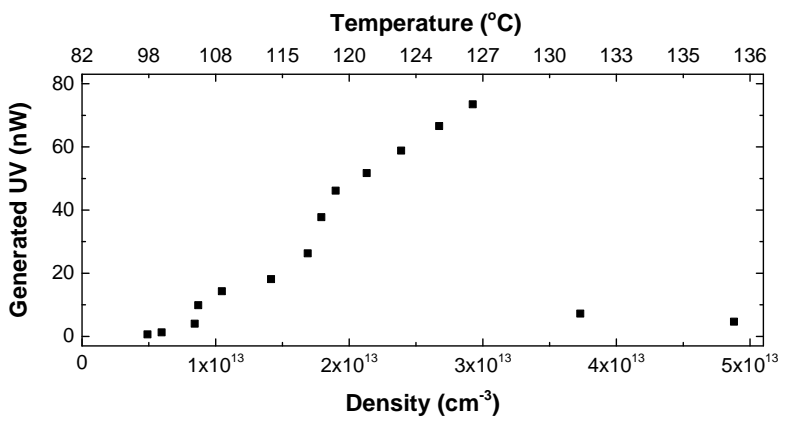

Fig. 6. Generated UV power as a function of atomic density. The vapor cell temperature is controlled externally to change the atomic density, and is given along the horizontal axis at the top, while the atomic density of the corresponding saturated vapor pressure is given along the axis at the bottom.

The density-dependence of the generated UV power is shown in Fig. 6, where both detunings and 780nm pump power are configured for maximum UV output at a fixed $515 \mathrm{~nm}$ pump power of $14 \mathrm{~mW}$. An approximately linear relationship is observed at lower densities. However, UV generation falls sharply to almost zero for densities larger than $3 \times 10^{-13} \mathrm{~cm}^{-3}$, which persists even with increasing $780 \mathrm{~nm}$ power and/or adjusting the laser detunings. This abrupt reduction may be due to ions created by collisions between Rydberg atoms [26, 27], which induce Stark shifts of atomic levels. Further investigation is needed to verify this assumption.

In conclusion, we have demonstrated the generation of deep UV light via FWM involving low lying Rydberg states. Despite multiple decay pathways from the upper Rydberg level $10 \mathrm{D}_{5 / 2}$, the dominant FWM process involves the most strongly dipolecoupled Rydberg level $11 \mathrm{P}_{3 / 2}$. This results in the generation of a nearly monochromatic UV beam, while other FWM pathways give rise to a much weaker nonlinearity and are thus strongly suppressed.

Besides being useful for up conversion to deep UV radiation, this scheme of optically pumping atomic media into low-lying Rydberg states may offer a possibility for building up a continuous wave (CW) THz source with high spectral brightness. This is especially interesting, given that there has been tremendous efforts [28-30] to develop compact, narrow-linewidth, high-power $\mathrm{THz}$ sources. Even though it is not possible to extract the $\mathrm{THz}$ radiation in our current apparatus, our theoretical simulations allow us to estimate the amount of $\mathrm{THz}$ radiation generated on the $10 \mathrm{D}_{5 / 2} \rightarrow 11 \mathrm{P}_{3 / 2}$ transition. We find that, under the current experimental conditions, the maximal power of the $\mathrm{THz}$ beam co-propagating with the other beams is of the order of 1 - 10 $\mu \mathrm{W}$ before the exit of the vapor cell. To facilitate extraction of the $\mathrm{THz}$ radiation, a new vapour cell with silicon output window is being constructed. To further increase the power of the generated UV or THz fields, a build-up cavity may be used. In summary, we believe that our experimental demonstration lays the ground work for further utilizing this scheme to generate coherent deep UV and $\mathrm{THz}$ radiations not easily accessible by other methods.

\section{ACKNOWLEDGMENTS}

The authors acknowledge the support by the National Research Foundation, Prime Minister's Office, Singapore and the Ministry of Education, Singapore under the Research Centres of Excellence programme.

\section{REFERENCES}

1. M. Lukin, P. Hemmer, and M. Scully, "Resonant nonlinear optics in phase-coherent media," (Academic Press, 2000), pp. 347 - 386.

2. M. Fleischhauer, A. Imamoglu, and J. P. Marangos, Reviews of modern physics 77, 633 (2005).

3. E. Arimondo, " $V$ coherent population trapping in laser spectroscopy," in "Progress in optics," , vol. 35 (Elsevier, 1996), pp. 257-354.

4. M. O. Scully, S.-Y. Zhu, and A. Gavrielides, Physical review letters 62 , 2813 (1989).

5. R. C. Pooser, A. M. Marino, V. Boyer, K. M. Jones, and P. D. Lett, Opt. Express 17, 16722 (2009).

6. B. Srivathsan, G. K. Gulati, B. Chng, G. Maslennikov, D. Matsukevich, and C. Kurtsiefer, Physical Review Letters 111, 123602 (2013).

7. A. Radnaev, Y. Dudin, R. Zhao, H. Jen, S. Jenkins, A. Kuzmich, and T. Kennedy, Nature Physics 6, 894 (2010).

8. G. Walker, A. Arnold, and S. Franke-Arnold, Physical review letters 108, 243601 (2012).

9. A. Zibrov, M. Lukin, L. Hollberg, and M. Scully, Physical Review A 65, 051801 (2002).

10. T. Meijer, J. D. White, B. Smeets, M. Jeppesen, and R. E. Scholten, Opt. Lett. 31, 1002 (2006).

11. A. M. Akulshin, R. J. McLean, A. I. Sidorov, and P. Hannaford, Opt. Express 17, 22861 (2009).

12. A. Vernier, S. Franke-Arnold, E. Riis, and A. S. Arnold, Opt. Express 18, 17020 (2010).

13. E. Brekke and L. Alderson, Opt. Lett. 38, 2147 (2013).

14. A. Akulshin, D. Budker, and R. McLean, Opt. Lett. 39, 845 (2014).

15. J. F. Sell, M. A. Gearba, B. D. DePaola, and R. J. Knize, Opt. Lett. 39, 528 (2014).

16. R. F. Offer, J. W. C. Conway, E. Riis, S. Franke-Arnold, and A. S. Arnold, Opt. Lett. 41, 2177 (2016).

17. F. Becerra, R. Willis, S. Rolston, and L. Orozco, Physical review A 78, 013834 (2008).

18. J. Schultz, S. Abend, D. Döring, J. Debs, P. Altin, J. White, N. Robins, and J. Close, Optics letters 34, 2321 (2009).

19. I. Beterov, I. Ryabtsev, D. Tretyakov, and V. Entin, Physical review A 79, 052504 (2009).

20. U. Sch $\tilde{A}^{1 / 4 n e m a n n}, H$. Engler, R. Grimm, M. Weidem $\tilde{A}^{1 / 4 l l e r, ~ a n d ~}$ M. Zielonkowski, Review of Scientific Instruments 70, 242 (1999).

21. A. Mohapatra, T. Jackson, and C. Adams, Physical review letters 98, 113003 (2007).

22. R. P. Abel, A. K. Mohapatra, M. G. Bason, J. D. Pritchard, K. J. Weatherill, U. Raitzsch, and C. S. Adams, Applied Physics Letters 94, 071107 (2009).

23. D. R. Skinner and R. E. Whitcher, Journal of Physics E: Scientific Instruments 5, 237 (1972).

24. J. M. Khosrofian and B. A. Garetz, Appl. Opt. 22, 3406 (1983).

25. J. Han, T. Vogt, C. Gross, D. Jaksch, M. Kiffner, and W. Li, Physical review letters 120, 093201 (2018).

26. D. Weller, A. Urvoy, A. Rico, R. Löw, and H. Kübler, Physical review A 94, 063820 (2016).

27. C. Wade, M. Marcuzzi, E. Levi, J. Kondo, I. Lesanovsky, C. Adams, and K. Weatherill, Nature Communication 9, 3567 (2018).

28. R. A. Lewis, Journal of Physics D: Applied Physics 47, 374001 (2014).

29. S. S. D. et al., Journal of Physics D: Applied Physics 50, 043001 (2017).

30. G. Liang, T. Liu, and Q. J. Wang, IEEE Journal of Selected Topics in Quantum Electronics 23, 1 (2017). 


\section{REFERENCES}

1. M. Lukin, P. Hemmer, and M. Scully, "Resonant nonlinear optics in phase-coherent media," (Academic Press, 2000), pp. 347 - 386.

2. M. Fleischhauer, A. Imamoglu, and J. P. Marangos, "Electromagnetically induced transparency: Optics in coherent media," Reviews of modern physics 77, 633 (2005).

3. $\quad$ E. Arimondo, "V coherent population trapping in laser spectroscopy," in "Progress in optics,", vol. 35 (Elsevier, 1996), pp. 257-354.

4. M. O. Scully, S.-Y. Zhu, and A. Gavrielides, "Degenerate quantum-beat laser: Lasing without inversion and inversion without lasing," Physical review letters 62, 2813 (1989).

5. R. C. Pooser, A. M. Marino, V. Boyer, K. M. Jones, and P. D. Lett, "Quantum correlated light beams from non-degenerate four-wave mixing in an atomic vapor: the $\mathrm{d} 1 \mathrm{and} \mathrm{d} 2 \mathrm{lines}$ of $85 \mathrm{rb}$ and $87 \mathrm{rb}$," Opt. Express 17, 16722-16730 (2009).

6. B. Srivathsan, G. K. Gulati, B. Chng, G. Maslennikov, D. Matsukevich, and C. Kurtsiefer, "Narrow band source of transform-limited photon pairs via four-wave mixing in a cold atomic ensemble," Physical Review Letters 111, 123602 (2013).

7. A. Radnaev, Y. Dudin, R. Zhao, H. Jen, S. Jenkins, A. Kuzmich, and T. Kennedy, "A quantum memory with telecom-wavelength conversion," Nature Physics 6, 894 (2010).

8. G. Walker, A. Arnold, and S. Franke-Arnold, "Trans-spectral orbital angular momentum transfer via four-wave mixing in rb vapor," Physical review letters 108, 243601 (2012).

9. A. Zibrov, M. Lukin, L. Hollberg, and M. Scully, "Efficient frequency up-conversion in resonant coherent media," Physical Review A 65, 051801 (2002).

10. T. Meijer, J. D. White, B. Smeets, M. Jeppesen, and R. E. Scholten, "Blue five-level frequency-upconversion system in rubidium," Opt. Lett. 31, 1002-1004 (2006).

11. A. M. Akulshin, R. J. McLean, A. I. Sidorov, and P. Hannaford, "Coherent and collimated blue light generated by four-wave mixing in $\mathrm{rb}$ vapour," Opt. Express 17, 22861-22870 (2009).

12. A. Vernier, S. Franke-Arnold, E. Riis, and A. S. Arnold, "Enhanced frequency up-conversion in rb vapor," Opt. Express 18, 17020-17026 (2010).

13. E. Brekke and L. Alderson, "Parametric four-wave mixing using a single cw laser," Opt. Lett. 38, 2147-2149 (2013).

14. A. Akulshin, D. Budker, and R. McLean, "Directional infrared emission resulting from cascade population inversion and four-wave mixing in $\mathrm{rb}$ vapor," Opt. Lett. 39, 845-848 (2014).

15. J. F. Sell, M. A. Gearba, B. D. DePaola, and R. J. Knize, "Collimated blue and infrared beams generated by two-photon excitation in rb vapor," Opt. Lett. 39, 528-531 (2014).

16. R. F. Offer, J. W. C. Conway, E. Riis, S. Franke-Arnold, and A. S. Arnold, "Cavity-enhanced frequency up-conversion in rubidium vapor," Opt. Lett. 41, 2177-2180 (2016).

17. F. Becerra, R. Willis, S. Rolston, and L. Orozco, "Nondegenerate fourwave mixing in rubidium vapor: The diamond configuration," Physical review A 78, 013834 (2008).

18. J. Schultz, S. Abend, D. Döring, J. Debs, P. Altin, J. White, N. Robins, and J. Close, "Coherent $455 \mathrm{~nm}$ beam production in a cesium vapor," Optics letters 34, 2321-2323 (2009).

19. I. Beterov, I. Ryabtsev, D. Tretyakov, and V. Entin, "Quasiclassical calculations of blackbody-radiation-induced depopulation rates and effective lifetimes of rydberg $n s, n p$, and $n d$ alkali-metal atoms with $n \leq 80$," Physical review A 79, 052504 (2009).

20. U. SchÃ $1 / 4$ nemann, H. Engler, R. Grimm, M. WeidemÃ $1 / 4$ ller, and M. Zielonkowski, "Simple scheme for tunable frequency offset locking of two lasers," Review of Scientific Instruments 70, 242-243 (1999).

21. A. Mohapatra, T. Jackson, and C. Adams, "Coherent optical detection of highly excited rydberg states using electromagnetically induced transparency," Physical review letters 98, 113003 (2007).

22. R. P. Abel, A. K. Mohapatra, M. G. Bason, J. D. Pritchard, K. J. Weatherill, U. Raitzsch, and C. S. Adams, "Laser frequency stabilization to excited state transitions using electromagnetically induced transparency in a cascade system," Applied Physics Letters 94, 071107
(2009).

23. D. R. Skinner and R. E. Whitcher, "Measurement of the radius of a high-power laser beam near the focus of a lens," Journal of Physics E: Scientific Instruments 5, 237 (1972).

24. J. M. Khosrofian and B. A. Garetz, "Measurement of a gaussian laser beam diameter through the direct inversion of knife-edge data," Appl. Opt. 22, 3406-3410 (1983).

25. J. Han, T. Vogt, C. Gross, D. Jaksch, M. Kiffner, and W. Li, "Coherent microwave-to-optical conversion via six-wave mixing in rydberg atoms," Physical review letters 120, 093201 (2018).

26. D. Weller, A. Urvoy, A. Rico, R. Löw, and H. Kübler, "Charge-induced optical bistability in thermal rydberg vapor," Physical review A 94, 063820 (2016).

27. C. Wade, M. Marcuzzi, E. Levi, J. Kondo, I. Lesanovsky, C. Adams, and K. Weatherill, "A terahertz-driven non-equilibrium phase transition in a room temperature atomic vapour," Nature Communication 9, 3567 (2018).

28. R. A. Lewis, "A review of terahertz sources," Journal of Physics D: Applied Physics 47, 374001 (2014).

29. S. S. D. et al., "The 2017 terahertz science and technology roadmap," Journal of Physics D: Applied Physics 50, 043001 (2017).

30. G. Liang, T. Liu, and Q. J. Wang, "Recent developments of terahertz quantum cascade lasers," IEEE Journal of Selected Topics in Quantum Electronics 23, 1-18 (2017). 